\title{
Dynamic response of a novel laminated glass panel using a \\ 2 transparent glass fiber-reinforced composite interlayer under blast loading
}

4 Hua Zhu, Sanjeev K. Khanna*

5 Mechanical and Aerospace Engineering Department, University of Missouri at Columbia, Columbia, Missouri

6 65201, United States

8 ABSTRACT

9 The novel laminated glass-composite panel consists of a transparent glass fiber-reinforced

10 polymer composite interlayer bonded to glass sheets. A numerical model has been developed to

11 simulate the dynamic response of the laminated glass-composite panel under blast loading. The

12 simulated dynamic response, in terms of the midpoint deflection correlates well with the

13 experiments conducted in a blast load simulator. Stress analysis of the laminated glass shows it

14 can survive under both medium intensity (peak pressure $\approx 4$ psi) and high intensity (peak

15 pressure $\approx 10$ psi or higher) blast.

16

17

18

19

23 Corresponding author. Tel.:+1 5738849109.

24 E-mail address: khannas@missouri.edu. 


\section{Introduction}

26 Terrorist bomb attacks and threats are on the rise all over the world [1-3]. One of the

27 significant effects of a blast is to damage glass-based windows in nearby buildings. The sharp

28 glass fragments produced from the damaged windows can lead to large casualties [4]. And blast

29 pressure entering the buildings through the damaged windows can cause additional injuries to the

30 occupants $[5,6]$. Hence, the need of mitigating the hazards caused by windows failure is urgent

31 [7-10]. One way to mitigate the damage is to use laminated glass, which is conventionally made

32 of one polyvinyl butyral (PVB) interlayer sandwiched between two glass sheets [5, 11-13], for

33 windows. Several experimental and analytical investigations have been carried out on studying

34 the response of the laminated glass with PVB interlayer under blast loading. Zhang et al. [10]

35 reported that PVB interlayer thickness can affect impact-resistant performance of laminated glass

36 window under impact loading. Increasing interlayer thickness can be effective in decreasing the

37 laminated glass penetration vulnerability. Hidallana-Gamage et al. [14] found the interlayer of

38 laminated glass has a major impact on the blast response of the laminated glass under severe

39 blast loads. Wei et al. [11] studied the behavior of laminated glass panels under simulated blast

40 loading. They found that bending stress mainly controls the response of a laminated plate under

41 blast loading and square panel is less blast resistant than a rectangular panel. Duser et al. [15]

42 analyzed the dynamic response of rectangular laminated glass panels under uniform lateral

43 pressure. Their analysis results showed that the loaded laminated glass panels responded in a

44 complex manner due to the large mismatch in stiffness of the two materials (glass/PVB). Hooper

45 et al. [16] studied the failure of laminated glasses under blast loading. Results showed that the

46 deflection profile of a laminated glass during a blast test could differ significantly from its static

47 deflection profile. 
Although laminated glass with PVB interlayer is widely used in the world [11, 15-19], it

49 still has some disadvantages, such as large thickness requirement for blast resistance, which

50 increases the production cost and installation cost. The thickness and weight can be potentially

51 reduced by replacing the PVB interlayer with a glass fiber-reinforced polymer composite

52 interlayer, because glass fiber-reinforced polymer composites have high strength to weight ratio

53 [20-22].

54 In this paper, a novel laminated glass using a transparent glass fiber-reinforced composite 55 interlayer has been successfully fabricated [23]. The transparency of the composite interlayer 56 was achieved by chemically matching the refractive index of the polyester matrix to that of glass

57 fibers. The optical property of the novel laminated glass was investigated using an ultraviolet58 visible (UV-VIS) spectrometer. The mechanical properties of the composite interlayer were 59 studied using an Instron universal testing machine. Field blast testing of the laminated glass was 60 done at the Engineering Research and Development Center (ERDC, US Army Corps of 61 Engineers Lab, Vicksburg, Mississippi) using a Blast Load Simulator (BLS). Real time loading 62 pressure and midpoint deflection of the laminated glass were recorded. A numerical model is 63 proposed to characterize the dynamic response of the fabricated laminated glass under blast 64 loading. The validity of the proposed numerical model has been proven by experimental results. 65 Laminated glass's failure analysis is also performed in this study using the stress analysis 66 approach.

67 


\section{Experimental Methods} 2.1 Transparent glass fiber-reinforced composite interlayer fabrication}

Polyester resin (Ashland Chemicals Co., USA) was mixed with $1.2 \%$ by weight of methyl

71 ethyl ketone peroxide (MEKP, Sigma-Aldrich Co., USA), an initiator, $4 \%$ by weight of divinyl

72 benzene (DV, Sigma-Aldrich Co., USA), a crosslinker and $0.03 \%$ by weight of cobalt (II) 2 -

73 ethylhexanoate (CE, Sigma-Aldrich Co., USA), an accelerator. By using this formulation, the

74 refractive index of the polyester matrix was chemically modified more closely to that of glass

75 fibers. All the above mentioned components were thoroughly mixed for 3-4 min by hand in a

76 plastic bucket. The mixture was set in a vacuum degassing chamber so as to allow air bubbles

77 inside it to escape by creating vacuum inside the chamber. After degassing, a small amount of

78 the mixture was first poured into a $3.2 \mathrm{~mm}$ deep mold so as to wet the base surface of the mold

79 which was made by placing aluminum frames on top of a polyvinyl chloride (PVC) plate with

80 Mylar sheet (Fig. 1). Then a layer of glass fiber cloth (Aerospace Composite Products Co., USA)

81 was placed in the mold and some more mixture was poured on top of the cloth. This procedure

82 was repeated 4 times, producing a composite of $3.2 \mathrm{~mm}$ thick with 5 layers of glass fiber cloth.

83 Finally, another PVC plate with Mylar sheet was laid on top of the frame, the top and bottom

84 plates were clamped with C-clamps. The clamped plates were erected sideways to let entrapped

85 air escape from the mold. The setup was left at room temperature $\left(\sim 20^{\circ} \mathrm{C}\right)$ for two days to ensure

86 the complete curing of the composite. 


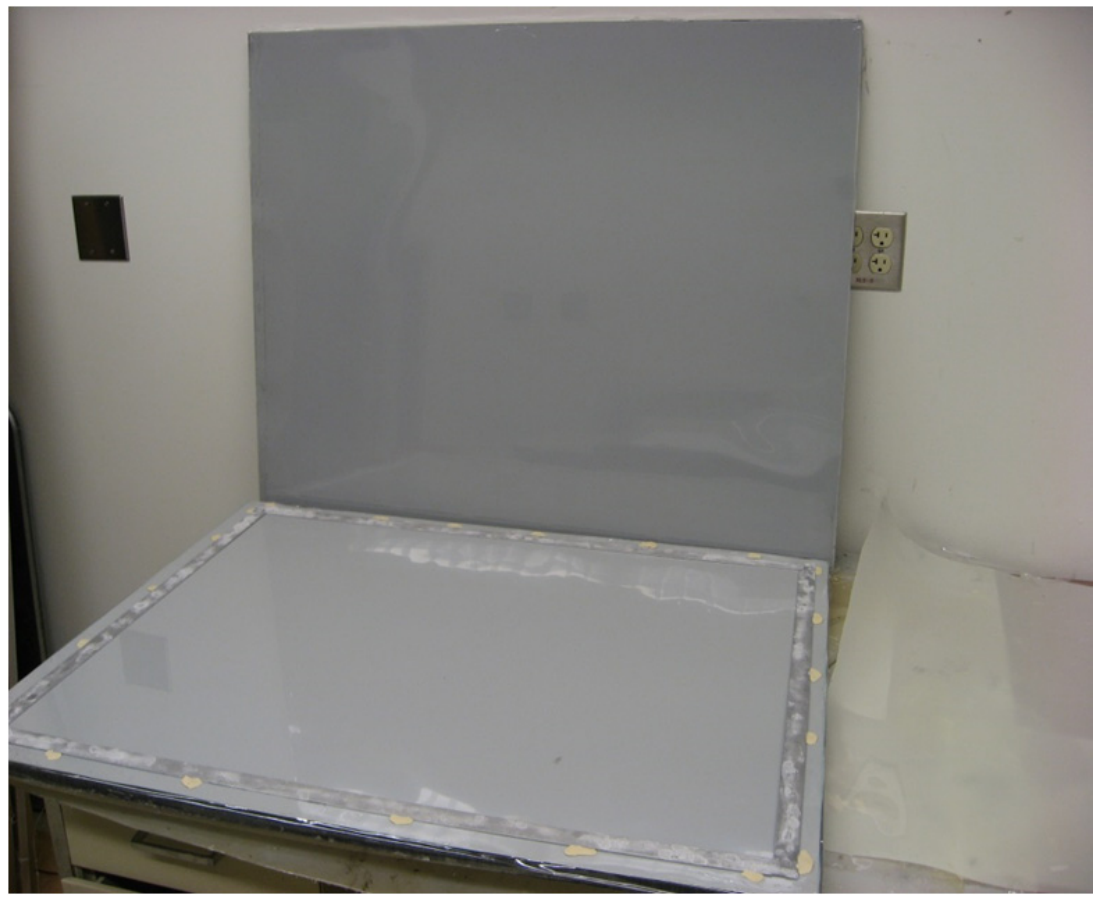

Figure 1 Mold for fabricating glass fiber-reinforced composite interlayer

\section{$93 \quad$ 2.2 Laminated glass panel fabrication}

94 The novel laminated glass panel was fabricated by sandwiching the glass fiber-reinforced

95 composite interlayer between two glass sheets (each of $3.2 \mathrm{~mm}$ or 0.125 in thick). The composite

96 interlayer and glass sheets were bonded using a two part polyurethane resin (SP\&S Co., USA).

97 The procedure for fabricating a laminated glass is as follows: firstly, a tempered glass sheet was

98 placed on a table and a very thin layer of polyurethane resin was uniformly spread on the glass

99 sheet. Secondly, the composite interlayer was placed on top of the glass sheet. Pressure was

100 applied to spread the resin and remove any entrapped air bubbles. Finally, a very thin layer of

101 polyurethane resin was uniformly spread on the composite interlayer and the second glass sheet

102 was placed on top of the composite interlayer. The setup was left at room temperature for at least

103 two days to ensure complete curing of the polyurethane adhesive. This process produced a

104 laminated panel of nominal thickness of about $10 \mathrm{~mm}$ or $0.375 \mathrm{in}$. 


\subsection{Composite interlayer's mechanical properties testing}

107 Since the interlayer is of a new transparent glass fiber reinforced polymer composite, its

108 mechanical properties were determined and used in modeling the mechanical response of the

109 panel to blast loading.

\subsubsection{Young's modulus and Poisson's ratio}

$112 \quad 250 \mathrm{~mm}$ long and $25 \mathrm{~mm}$ wide strips were cut from the fabricated composite sheets. The

113 strips were machined to ensure that they were straight and had smooth edges. Tensile tests were 114 performed at room temperature by using a servo-hydraulic Instron 8800 universal testing

115 machine with a fast track digital controller and a $10 \mathrm{kN}$ load cell, at a crosshead speed of $1162 \mathrm{~mm} / \mathrm{min}$. For the measurement of strains, strain gages (Vishary Precision Inc., USA) were

117 attached on the specimens in both longitudinal and lateral directions. During the test, loads and 118 strains were recorded by computer. These data were used to find Young's modulus $(E)$ and 119 Poisson's ratio $\left(v_{12}\right)$ of the composite based on ASTM standard D3039 [24].

\subsubsection{Shear modulus}

$122 \quad 250 \mathrm{~mm}$ long and $25 \mathrm{~mm}$ wide $\pm 45^{\circ}$ strips were cut from the fabricated composite sheets.

123 Strain gages were also attached on the specimens in both longitudinal and lateral directions. The $124 \pm 45^{\circ}$ specimens were loaded in tension while recording load and strain data. These data were 125 used to calculate shear modulus of the composite based on ASTM standard D3518 [25]. 


\section{$127 \quad 2.4$ Optical property testing}

128 The light transmittance of the fabricated laminated glass in the thickness direction was 129 measured over a wavelength range of 190 to $900 \mathrm{~nm}$ by using a UV-VIS (ultraviolet-visible)

130 spectrometer (UV 240PC, Shimadzu Co., Japan). The resolution of the equipment is $1 \mathrm{~nm}$ within 131 the measured wavelength range. All the measurements were done at the room temperature $132\left(\sim 20^{\circ} \mathrm{C}\right)$.

133

\section{$134 \quad 2.5$ Blast resistance testing}

135 A blast is a sudden release of stored energy. When a blast happens, rapid expansion of 136 energy resulting from the blast gives rise to a wave of compressed air which is called shock 137 front. The shock front travels radially in air in all directions. As the shock front moves, the shock 138 front releases energy to surrounding air and the overpressure of the shock front decreases. When 139 the pressure of the shock front drops below the atmospheric pressure, surrounding air gives 140 energy to the shock front and the pressure of the shock front finally returns to the atmospheric 141 pressure [26]. The whole process is shown schematically in Fig. 2 [11]. 


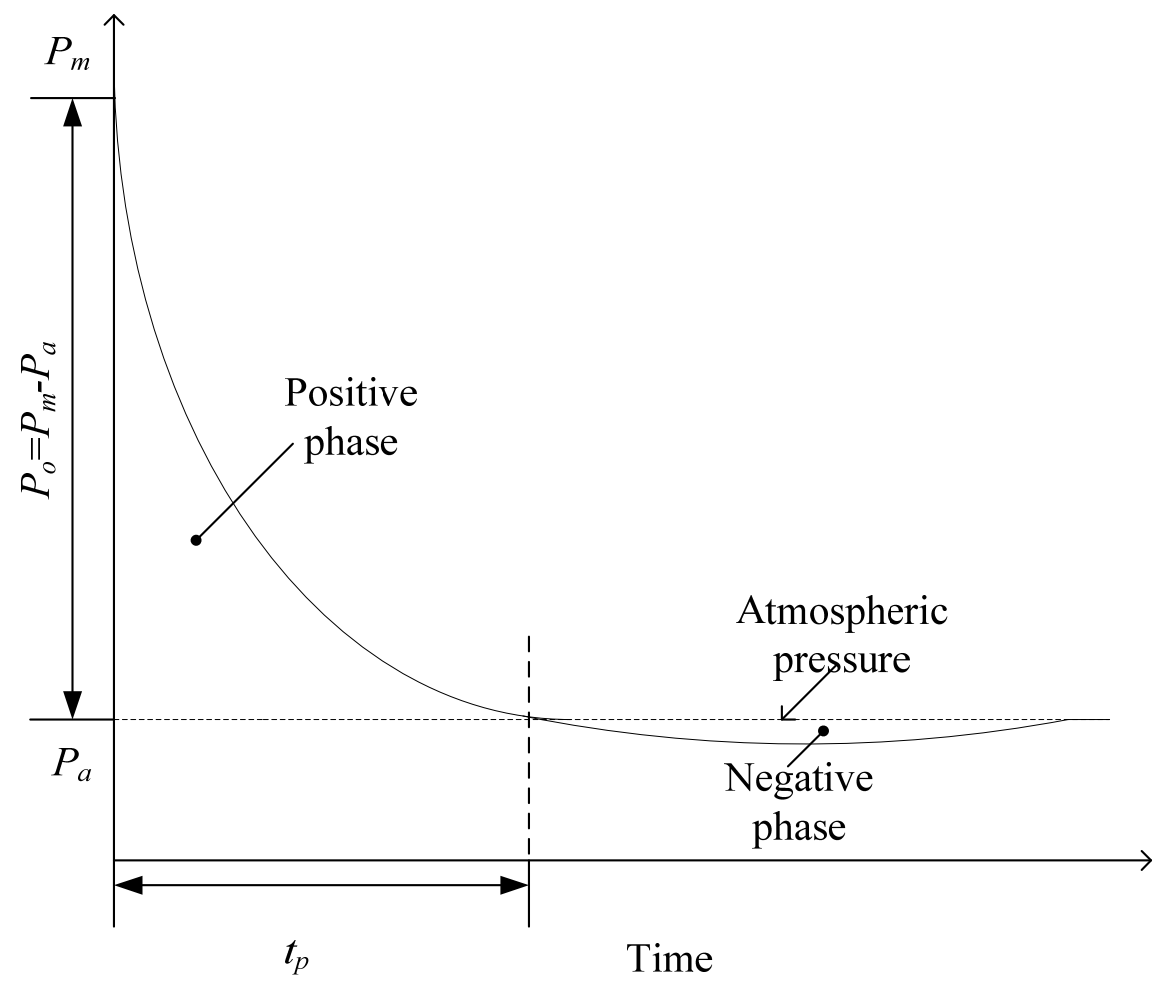

145 The pressure-time curve shown in Fig. 2 can be described using the following equation [27]

$$
P(t)=P_{o}\left(1-t / t_{p}\right) \mathrm{e}^{-\alpha t / t_{p}}
$$

149 where $P(t)$ is the blast pressure at time $t, P_{o}$ is the peak pressure, $\alpha$ is a constant and $t_{p}$ is the 150 positive pressure duration time. According to references [27, 28], the key parameters of a blast 151 are: peak pressure $P_{o}$, constant $\alpha$ and positive pressure duration time $t_{p}$.

152 Field blast loading experiments were done at the Engineering Research and Development 153 Center (ERDC, US Army Corps of Engineers Lab, Vicksburg, Mississippi) using a Blast Load 154 Simulator (BLS). The installation of the laminated glass inside the BLS is shown in Fig. 3. The 
155 thickness of the laminated glass was around $9.5 \mathrm{~mm}$ (a $1 / 8$ inch $(3.2 \mathrm{~mm})$ thick transparent 156 composite interlayer laminated to two $1 / 8$ inch $(3.2 \mathrm{~mm})$ thick tempered glass sheets). The length 157 and width of the laminated glass were $0.89 \mathrm{~m}$ and $0.59 \mathrm{~m}$, respectively. Sample gages were 158 attached to the laminated glass to record the pressure loading history and the midpoint deflection 159 history during the blast.

160

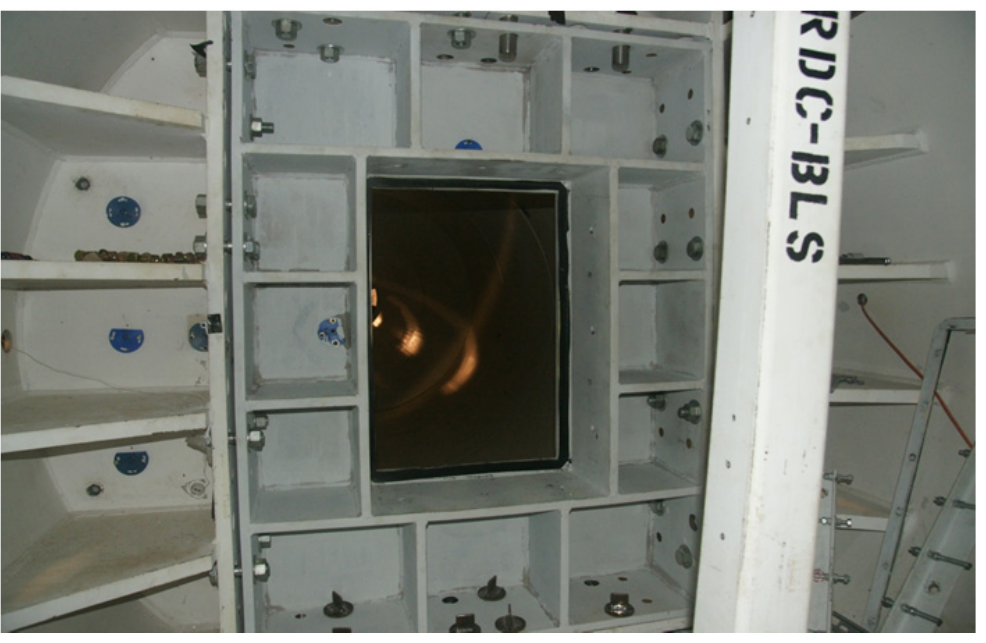

Figure 3 Laminated glass installation inside the BLS

164

\section{Experimental results}

\subsection{Mechanical properties of the composite interlayer}

168 Table 1, it can be seen that Young's modulus of the composite interlayer is $7.67 \mathrm{GPa}$ which is 169 much higher than that of the neat polyester ( 3 GPa) [29]. Poisson's ratio and shear modulus of 170 the composite are 0.33 and $2.14 \mathrm{GPa}$, respectively. 


\begin{tabular}{lccc}
\hline & $\begin{array}{c}\text { Young's modulus } \\
(E, \mathrm{GPa})\end{array}$ & $\begin{array}{c}\text { Poisson's } \\
\text { ratio }\left(v_{12}\right)\end{array}$ & $\begin{array}{c}\text { Shear modulus } \\
(\tau, \mathrm{GPa})\end{array}$ \\
\hline $\begin{array}{l}\text { Fiber-reinforced } \\
\text { composite }\end{array}$ & 7.67 & 0.33 & 2.14 \\
\hline
\end{tabular}

173

\section{$174 \quad 3.2$ Optical property of the novel laminated glass}

175 Fig. 4 shows the light transmittance of the fabricated laminated glass over a wavelength 176 range of $190-900 \mathrm{~nm}$. The light transmittance of the laminated glass is above $60 \%$ over a 177 wavelength range of 482 to $900 \mathrm{~nm}$. The highest transmittance is $84.4 \%$ when the wavelength is $178577 \mathrm{~nm}$. This result means the laminated glass has good transparency in the visible light range.

179 Similar results have been reported by Olsen et al. [30] and Iba et al. [31].

180 Appearance of distant objects through the laminated glass is shown in Fig. 5. Through the

181 laminated glass, the outside view can be clearly observed, which shows the fabricated laminated 182 glass has good transparency though there is a slight haziness. This result coincides with the light 183 transmittance spectra analysis result shown above.

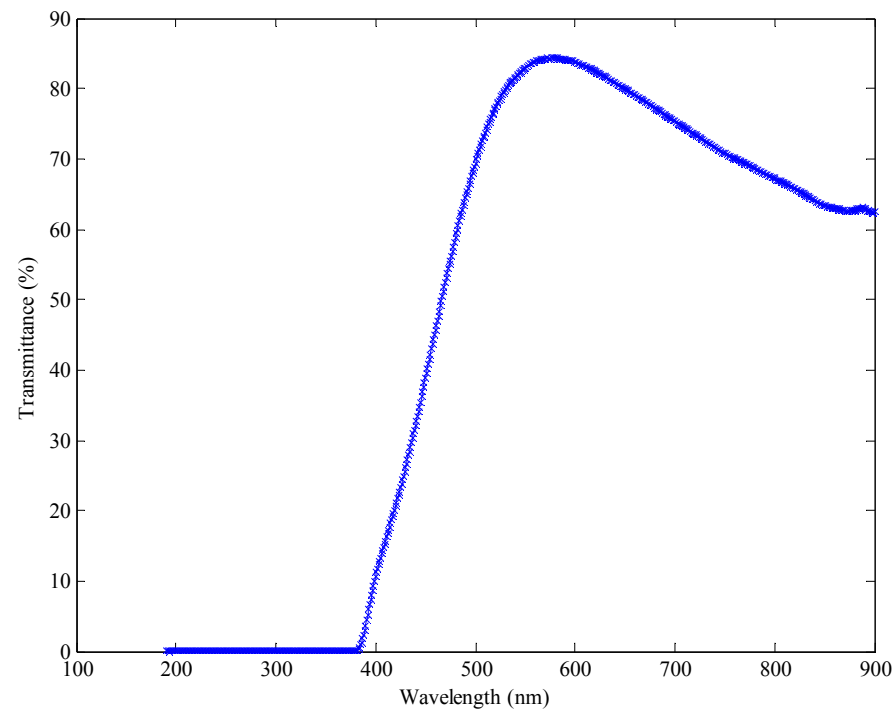




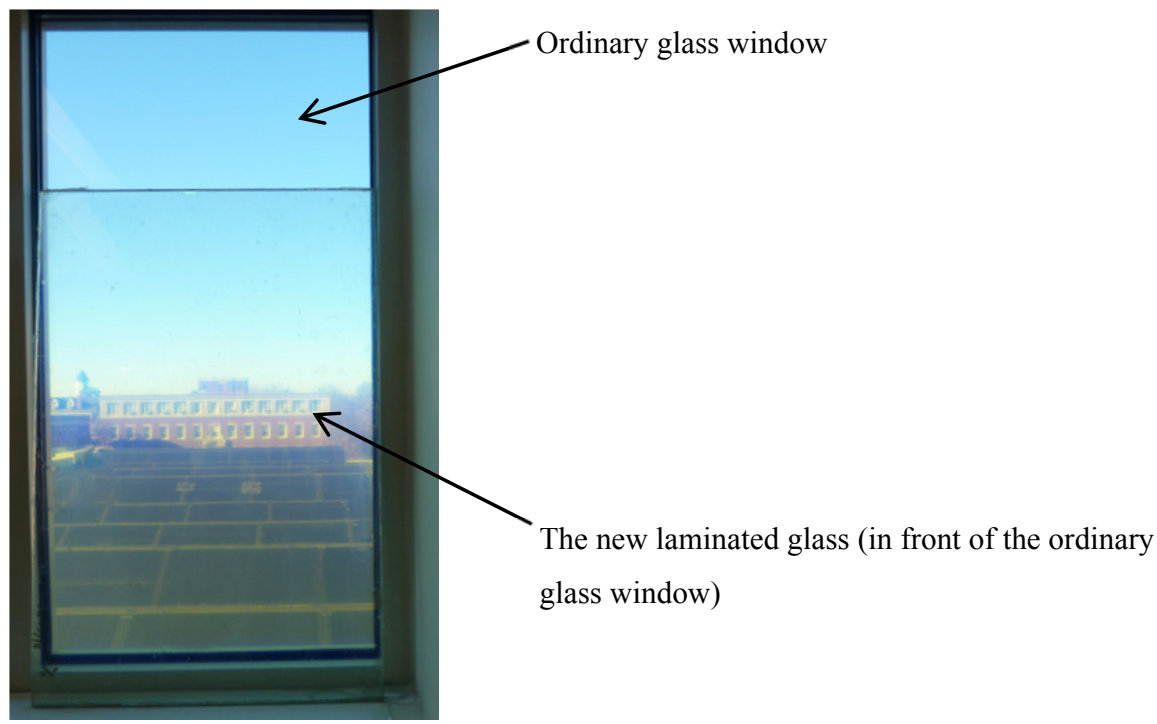

\subsection{Blast resistance testing results}

Both medium and high intensity blast loading tests were done at ERDC. The pressure-time

192 curve of the medium intensity blast test conducted in a blast load simulator is shown in Fig. 6.

193 The key parameters of the blast are obtained by curve fitting the initial pressure phase using

194 equation (1). The result of fitting is: peak overpressure $P_{o}=5.14 \mathrm{psi}(35.4 \mathrm{kPa})$, constant $195 \alpha=0.1011$, and positive pressure duration time $t_{p}=11.3 \mathrm{~ms}$.

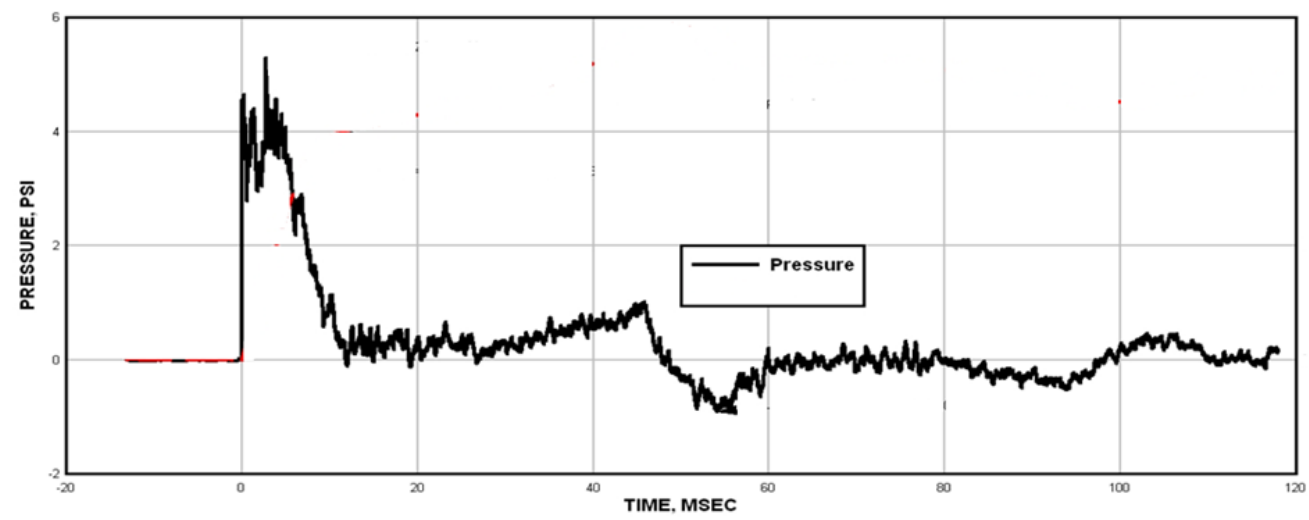

Figure 6 Pressure-time curve of the medium intensity blast 
Fig. 7 shows the midpoint deflection of the laminated glass under the medium intensity 201 blast shown in Fig. 6. It can be seen that the maximum deflection is about 0.415 inch $(10.5 \mathrm{~mm})$ 202 and appears in the first deflection peak region.

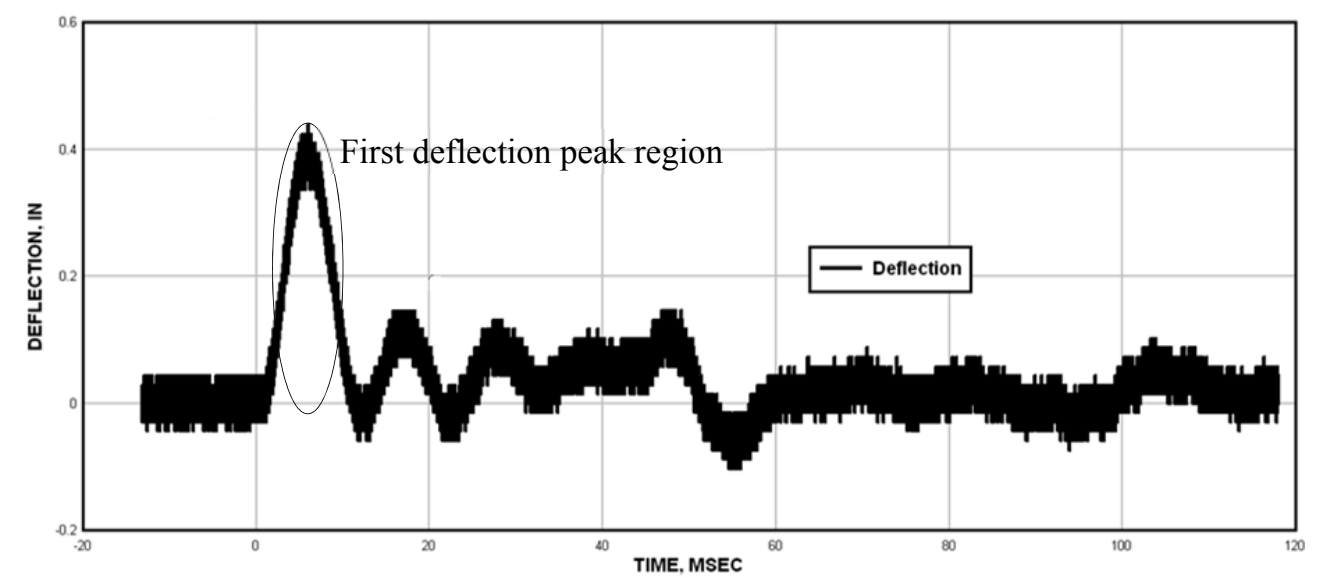

Figure 7 Midpoint deflection of the laminated glass under the medium intensity blast

210 the blast are: peak overpressure $P_{o}=13.22 \mathrm{psi}(91.1 \mathrm{kPa})$, constant $\alpha=0.2744$, positive pressure 211 duration time $t_{p}=15.4 \mathrm{~ms}$.

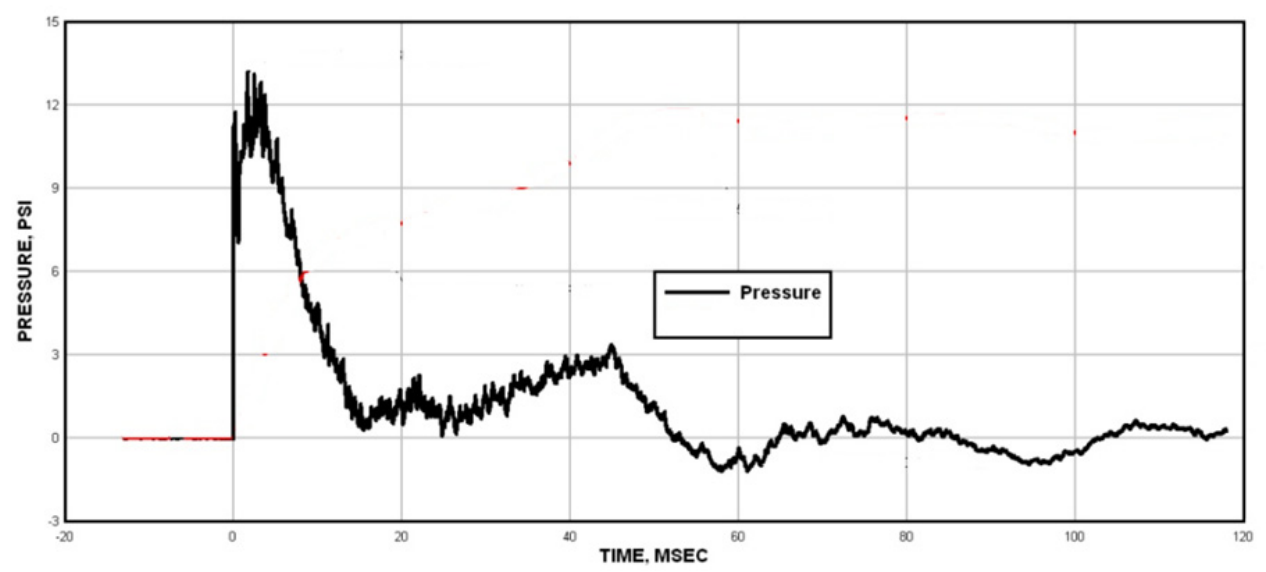

Figure 8 Pressure-time curve of the high intensity blast 
Fig. 9 shows the midpoint deflection of the laminated glass under the high intensity blast

217 shown in Fig. 8. The maximum deflection is around 0.99 inch $(25.1 \mathrm{~mm})$ and also appears in the 218 first deflection peak region.

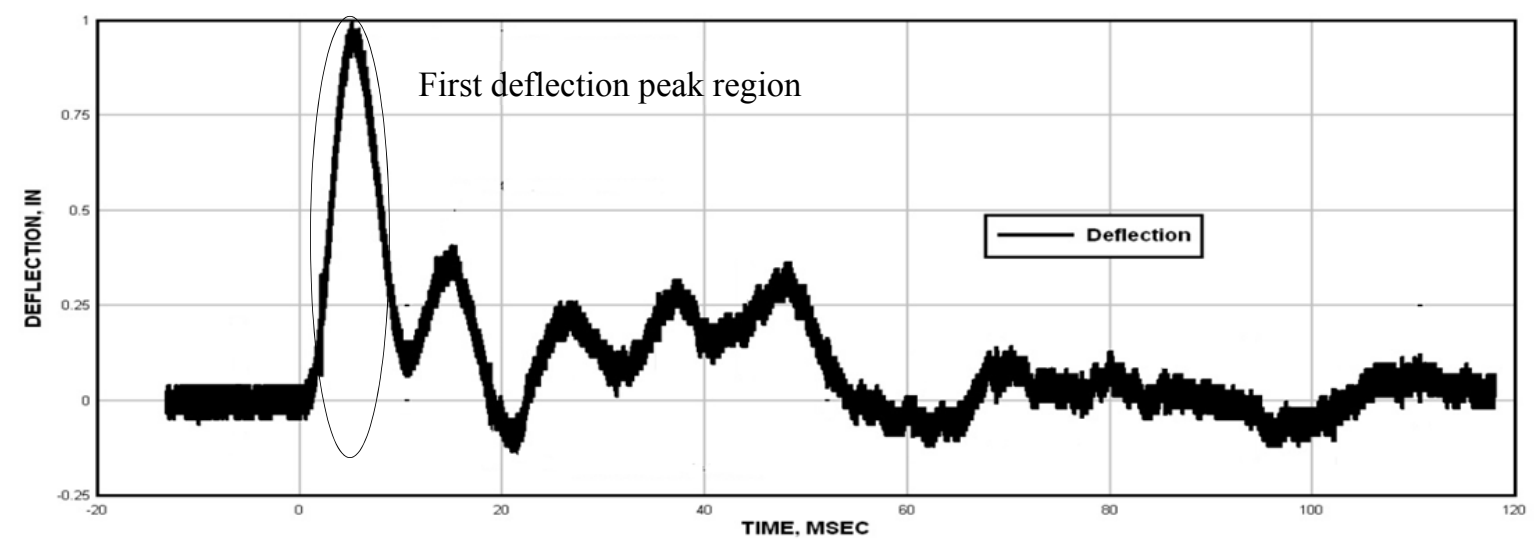

Figure 9 Midpoint deflection of the laminated glass under the high intensity blast

223 Field experiments are important for understanding the dynamic response of the laminated 224 glass under blast loading. Besides field testing, model-based analysis can also be used to study 225 the dynamic response of the laminated glass under blast loading. In this study, a numerical model 226 is proposed to characterize the dynamic response of the fabricated laminated glass under blast 227 loading. glass panel under blast loading

232 Fig. 10. 
Use Hamilton's principle to obtain the equation of motion of the laminated glass

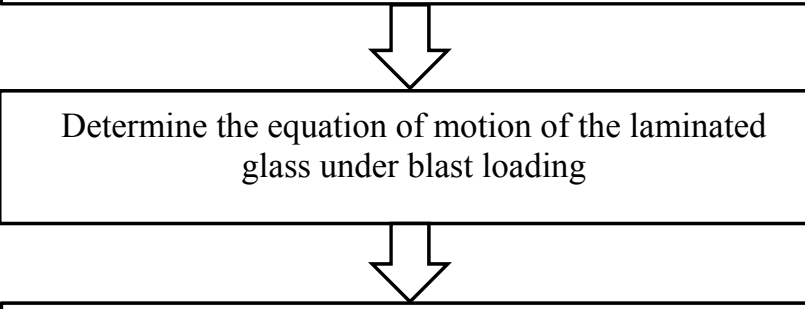

Choose a function to describe the deflection $w$ by considering the simply supported boundary condition

The detailed derivations are listed below.

$$
\int_{t_{1}}^{t_{2}}(\delta T-\delta \Pi+\delta W) d t=0
$$

255 where $T$ is the kinetic energy of the laminated glass, $\Pi$ is the strain energy of the laminated glass 256 and $W$ is the work done by external load(s).

257 For the laminated glass, kinetic energy $T$ can be calculated using the following equation [33] 258 
$259 \quad T=\frac{1}{2} M \int_{0}^{a} \int_{0}^{b}\left(\frac{\partial w}{\partial t}\right)^{2} d x d y$

261 where $M$ is the unit area mass of the laminated glass and is equal to $\rho_{o} h_{o}+\rho_{c} h_{c}+\rho_{i} h_{i}$, and $\rho_{\mathrm{o}}$,

$262 \rho_{\mathrm{c}}$ and $\rho_{\mathrm{i}}$ are the density of the outer glass sheet, the composite interlayer and the inner glass

263 sheet, respectively. $\rho_{\mathrm{o}}=\rho_{\mathrm{i}}=2600 \mathrm{~kg} / \mathrm{m}^{3}$ and $\rho_{\mathrm{c}}=1200 \mathrm{~kg} / \mathrm{m}^{3} . h_{\mathrm{o}}, h_{c}$, and $h_{i}$ are the thickness of

264 the outer glass sheet, the composite interlayer and the inner glass sheet, respectively. In this

265 study, $h_{\mathrm{o}}=h_{c}=h_{i}=3.2 \mathrm{~mm}, w$ is the transverse (thickness direction) deflection of the laminated

266 glass.

267 Strain energy $\Pi$ can be calculated using the following equation [33]

268

269

$$
\Pi=\frac{1}{2} \iiint_{V}\left(\sigma_{x} \varepsilon_{x}+\sigma_{y} \varepsilon_{y}+\tau_{x y} \gamma_{x y}\right) d x d y d z
$$

272 can be expressed by the transverse deflection, $w$, as

273

$$
\varepsilon_{x}=-z \frac{\partial^{2} w}{\partial x^{2}}, \quad \varepsilon_{y}=-z \frac{\partial^{2} w}{\partial y^{2}}, \quad \gamma_{x y}=-2 z \frac{\partial^{2} w}{\partial x \partial y}
$$




$$
\Pi_{\text {comp }}=\frac{1}{2} \iiint_{V}\left(\frac{E_{c} \varepsilon_{x} \varepsilon_{x}}{1-v_{c}^{2}}+\frac{v_{c} E_{c} \varepsilon_{y} \varepsilon_{x}}{1-v_{c}^{2}}+\frac{v_{c} E_{c} \varepsilon_{x} \varepsilon_{y}}{1-v_{c}^{2}}+\frac{E_{c} \varepsilon_{y} \varepsilon_{y}}{1-v_{c}^{2}}+G_{c} \gamma_{x y} \gamma_{x y}\right) d x d y d z
$$

Figure 11 Schematic diagram of the laminated glass

For the orthotropic composite interlayer, $\sigma_{x}=\frac{E_{c}}{1-v_{c}^{2}} \varepsilon_{x}+\frac{v_{c} E_{c}}{1-v_{c}^{2}} \varepsilon_{y}, \sigma_{y}=\frac{v_{c} E_{c}}{1-v_{c}^{2}} \varepsilon_{x}+\frac{E_{c}}{1-v_{c}^{2}} \varepsilon_{y}$ and $\tau_{x y}=G_{c} \gamma_{x y}$, where $E_{c}, G_{c}$ and $v_{c}$ are Young's modulus, shear modulus and Poisson's ratio of the composite interlayer, respectively. It should be noted that the mechanical properties of the glass fiber-reinforced composite, such as Young's modulus and shear modulus, are influenced by strain rate [35]. However, in this analysis, since strain rate was not measured in field testing, the strain rate effect is not considered. So $E_{c}, G_{c}$ and $v_{c}$ values used here are the values obtained from previous quasi-static mechanical tests (Table 1). Substituting $\sigma_{x}, \sigma_{y}$ and $\tau_{x y}$ expressions into equation (4), the strain energy of the composite interlayer is

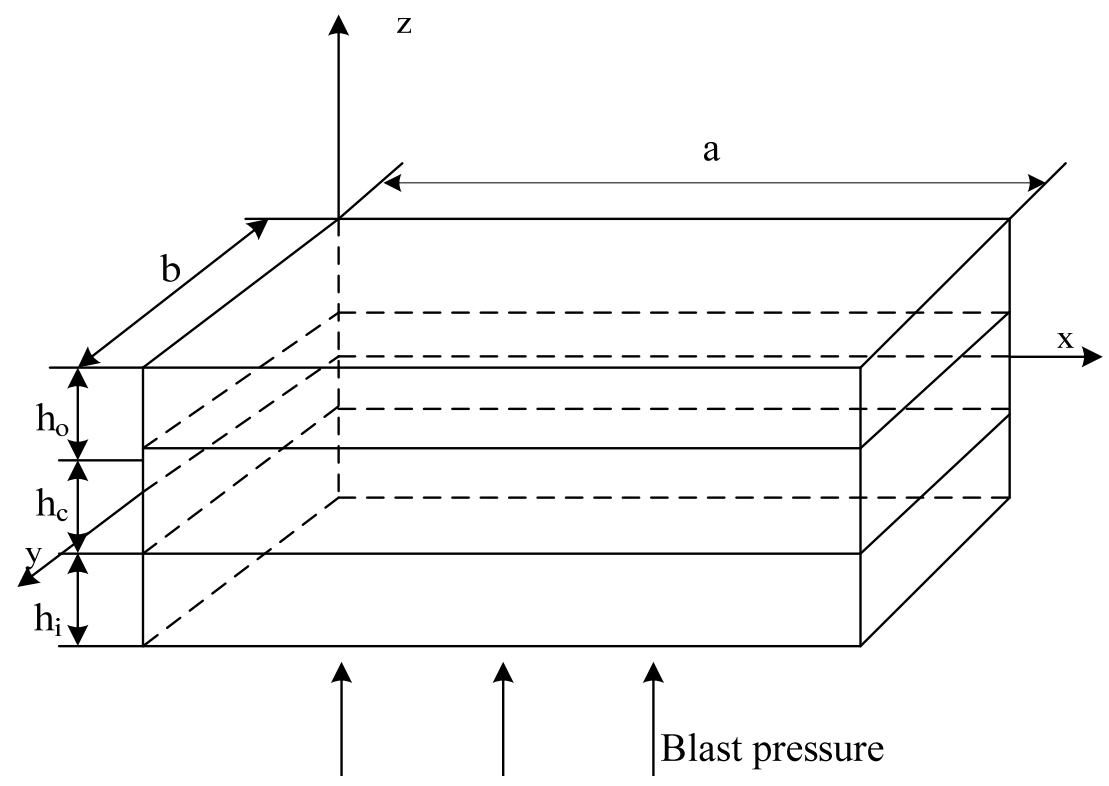


Integrating equation (6) through the transverse direction (thickness direction, $\mathrm{z}$ direction) results

291 in

292

293

$$
\Pi_{\text {comp }}=\frac{1}{2} \int_{0}^{a} \int_{0}^{b}\left[\begin{array}{l}
\frac{E_{c} h_{c}^{3}}{12\left(1-v_{c}^{2}\right)}\left(\frac{\partial^{2} w}{\partial x^{2}}\right)^{2}+\frac{E_{c} h_{c}^{3}}{12\left(1-v_{c}^{2}\right)}\left(\frac{\partial^{2} w}{\partial y^{2}}\right)^{2} \\
+\frac{2 v_{c} E_{c} h_{c}^{3}}{12\left(1-v_{c}^{2}\right)}\left(\frac{\partial^{2} w}{\partial x^{2}}\right)\left(\frac{\partial^{2} w}{\partial y^{2}}\right)+\frac{4 G_{c} h_{c}^{3}}{12}\left(\frac{\partial^{2} w}{\partial x \partial y}\right)^{2}
\end{array}\right] d x d y
$$

294

295 where $a$ is the length of the laminated glass, $b$ is width of the laminated glass. In this study, $a=$ $0.89 \mathrm{~m}$ and $b=0.59 \mathrm{~m}$.

297

Similarly, the strain energy of the outer and inner glass sheets is

298

299

$$
\begin{aligned}
\Pi_{\text {glass }}= & \frac{1}{2}\left\{\frac{2 E_{g}}{3\left(1-v_{g}^{2}\right)}\left[h_{\mathrm{g}}^{3}+\frac{3 h_{c}^{2} h_{g}}{4}+\frac{3 h_{g}^{2} h_{c}}{2}\right]\right\} \\
& \int_{0}^{a} \int_{0}^{b}\left[\left(\frac{\partial^{2} w}{\partial x^{2}}\right)^{2}+\left(\frac{\partial^{2} w}{\partial y^{2}}\right)^{2}+2 v_{g} \frac{\partial^{2} w}{\partial x^{2}} \frac{\partial^{2} w}{\partial y^{2}}+2\left(1-v_{g}\right)\left(\frac{\partial^{2} w}{\partial x \partial y}\right)^{2}\right] d x d y
\end{aligned}
$$

302 where $h_{g}$ is the thickness of one glass sheet (inner or outer layer glass sheet), $E_{g}$ is Young's

303 modulus of glass, $v_{g}$ is Poisson's ratio of glass, In this study, tempered glass is used, for which $E_{g}$

$304=68 \mathrm{GPa}, v_{g}=0.2$. Summing the strain energy of the composite interlayer and glass sheets, the 305 total strain energy of the laminated glass is 


$$
\begin{gathered}
\Pi=\frac{1}{2} \int_{0}^{a} \int_{0}^{b}\left[A\left(w_{x x}\right)^{2}+A\left(w_{y y}\right)^{2}+B w_{x x} w_{y y}+C\left(w_{x y}\right)^{2}\right] d x d y \\
A=\frac{2 E_{g}}{3\left(1-v_{g}^{2}\right)}\left[h_{g}^{3}+\frac{3 h_{c}^{2} h_{g}}{4}+\frac{3 h_{g}^{2} h_{c}}{2}\right]+\frac{E_{c} h_{c}^{3}}{12\left(1-v_{c}^{2}\right)} \\
B=2 v\left\{\frac{2 E_{g}}{3\left(1-v_{g}^{2}\right)}\left[h_{g}^{3}+\frac{3 h_{c}^{2} h_{g}}{4}+\frac{3 h_{g}^{2} h_{c}}{2}\right]\right\}+\frac{2 v_{c} E_{c} h_{c}^{3}}{12\left(1-v_{c}^{2}\right)} \\
C=2(1-v)\left\{\frac{2 E_{g}}{3\left(1-v_{g}^{2}\right)}\left[h_{g}^{3}+\frac{3 h_{c}^{2} h_{g}}{4}+\frac{3 h_{g}^{2} h_{c}}{2}\right]\right\}+\frac{4 G_{c} h_{c}^{3}}{12}
\end{gathered}
$$

308

309

310 where $w_{k l}$ means a differentiation with respect to variable $k$ and variable $l$.

$$
W=\int_{0}^{a} \int_{0}^{b} P(t) w d x d y
$$

315 where $P(t)$ is the instantaneous blast pressure and can be described by equation (1).

$$
\int_{t}^{t_{2}} \int_{0}^{a} \int_{0}^{b}\left\{-M \ddot{w} \delta w-\left(A w_{x x x x}+A w_{y y y y}+(B+C) w_{x x y y}\right) \delta w+P(t) \delta w\right\} d x d y d t=0
$$

320 where $\ddot{w}$ means a differentiation with respect to time. From equation (11), the equation of

321 motion of the laminated glass is obtained as 


$$
M \ddot{w}+A\left(w_{x x x x}+w_{y y y y}\right)+(B+C) w_{x x y y}=P(t)
$$

326 The laminated glass in this study is considered as simply supported. So the boundary conditions 327 are

$$
w=0, w_{x x}=0, \quad \text { at } x=0, a,
$$

$$
w=0, w_{y y}=0, \quad \text { at } y=0, b,
$$

331 The initial condition is

332

$$
w(x, y, 0)=0, \quad \dot{w}(x, y, 0)=0
$$

335 In order to solve equation (12), an approximate function is chosen for $w$ by considering the 336 simply supported boundary conditions $[11,33,36,37]$

$$
w(x, y, t)=\sum_{m=1}^{\infty} \sum_{n=1}^{\infty} \psi \sin \left(\frac{m \pi x}{a}\right) \sin \left(\frac{n \pi y}{b}\right)
$$

340 where $\psi$ is a unknown time dependent function. Here, for calculation convenience, $m$ and $n$ are

341 both simplified to 1 . Substituting equation (13) into equation (12) and applying Galerkin method,

342 the equation of motion of the laminated glass is changed to 


$$
\frac{\left(a\left(\frac{A \pi^{6} \psi}{4}-4 b^{4} P_{o}\left(1-t / t_{p}\right) \mathrm{e}^{-\alpha t / t_{p}}+\frac{M \pi^{2} \ddot{\psi} b^{4}}{4}\right)\right)}{\pi^{2} b^{3}}+\frac{a^{2}\left(\frac{B \pi^{6} \psi b^{2}}{4}+\frac{C \pi^{6} \psi b^{2}}{4}\right)+\frac{A \pi^{6} \psi b^{4}}{4}}{\pi^{2} a^{3} b^{3}}=0
$$

347 Rearranging equation (14) and the following equation is obtained

$$
\ddot{\psi}=\frac{4}{M \pi^{2} b^{4}}\left(-\frac{A \pi^{6} \psi}{4}+4 b^{4} P_{o}\left(1-t / t_{p}\right) \mathrm{e}^{-\alpha t / t_{p}}-\frac{a^{2}\left(\frac{B \pi^{6} \psi b^{2}}{4}+\frac{C \pi^{6} \psi b^{2}}{4}\right)+\frac{A \pi^{6} \psi b^{4}}{4}}{a^{4}}\right)
$$

Equation (15) is a nonlinear ordinary differential equation and can be solved using Runge-Kutta method. After obtaining function $\psi$, the transverse deflection $w$ can be calculated using equation (13).

\section{Comparison between numerical and experimental results}

Fig. 12 shows the predicted maximum deflection state of the laminated glass under the

357 medium intensity blast loading. Fig. 13 shows the predicted maximum deflection state of the

358 laminated glass under the high intensity blast loading. In Fig. 12 and 13, the laminated glass is

359 simplified to a zero-thickness plate. From Fig. 12 and 13, it can be seen that the maximum

360 deflection occurs at the midpoint. At the position close to the edge of the laminated glass, the

361 deflection decreases. This prediction correspnds fairly well with the experimental results. 


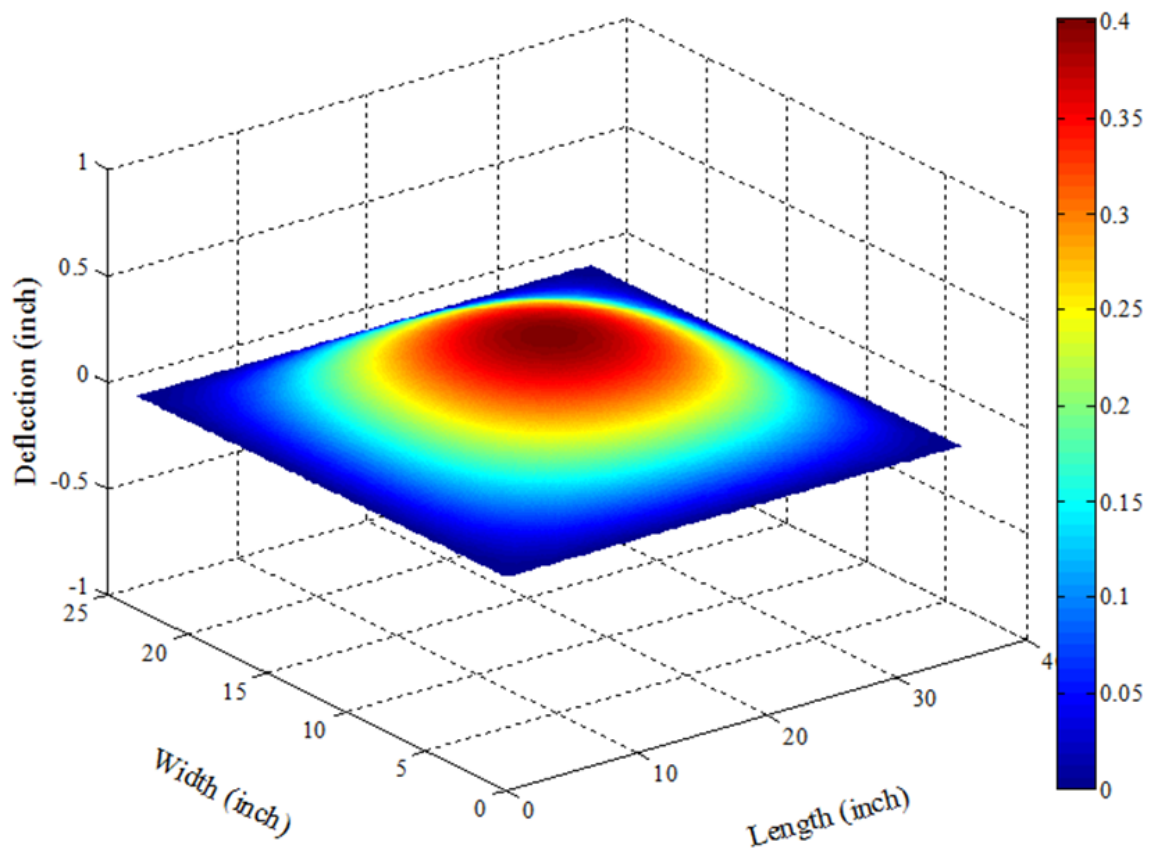

Figure 12 The maximum deflection state of the laminated 


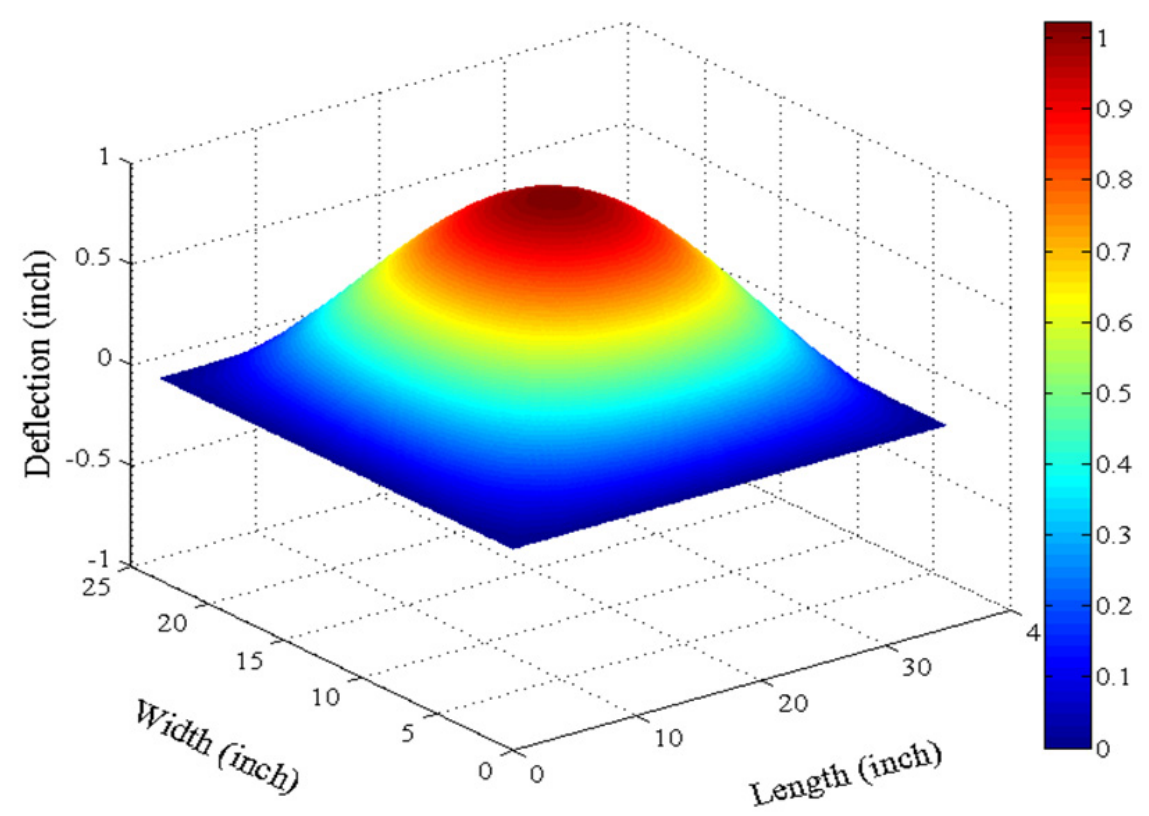

Figure 13 The maximum deflection state of the laminated glass under the high intensity blast loading

From Fig. 7 and 9, it can be seen that the maximum deflection, which is an important 379 criterion for evaluating the blast resistance of the fabricated laminated glass, occurs in the first 380 deflection peak region. Therefore, the knowledge of the deflection history in the first peak region 381 is important and is simulated by the developed numerical model. Fig. 14 shows the predicted 382 midpoint deflection history in the first deflection peak region. The predicted maximum 383 deflection is 0.42 inch $(10.6 \mathrm{~mm})$ and the predicted positive deflection duration time is $7.8 \mathrm{~ms}$.

384 The experimentally measured midpoint deflection history (the circled region in Fig. 7) is also 385 plotted in Fig. 14. The experimentally measured maximum deflection value is 0.415 inch (10.5 $386 \mathrm{~mm}$ ) and the measured duration time is $8.3 \mathrm{~ms}$. The discrepancy between the numerical result 387 and the experimental result may be caused by the neglect of the composite interlayer's plastic 388 deformation and the strain rate effect on mechanical properties in the modeling. It can be 
observed from Fig. 14 that for the medium intensity blast loading, the predicted result matches

390 well with the experimentally measured result.

391 Similarly, for the high intensity blast loading, the predicted midpoint deflection history in

392 the first deflection peak region is compared in Fig. 15 with the experimentally measured result.

393 The predicted maximum deflection is 1.03 inch $(26.1 \mathrm{~mm})$ and the predicted positive deflection

394 duration time is $8.1 \mathrm{~ms}$. The experimentally measured maximum deflection value is 0.99 inch

$395(25.2 \mathrm{~mm})$ and the measured duration time is $9.4 \mathrm{~ms}$. Fig. 15 shows that for the high intensity

396 blast loading, the predicted result also matches fairly well with the experimentally measured

397 result. Considering the good match of the numerically predicted results and the experimentally

398 measured results under medium and high intensity blast loading, it can be concluded that the

399 developed numerical model is valid for predicting the dynamic reponse of the laminated glass

400 under both medium and high intensity blast loading.

401

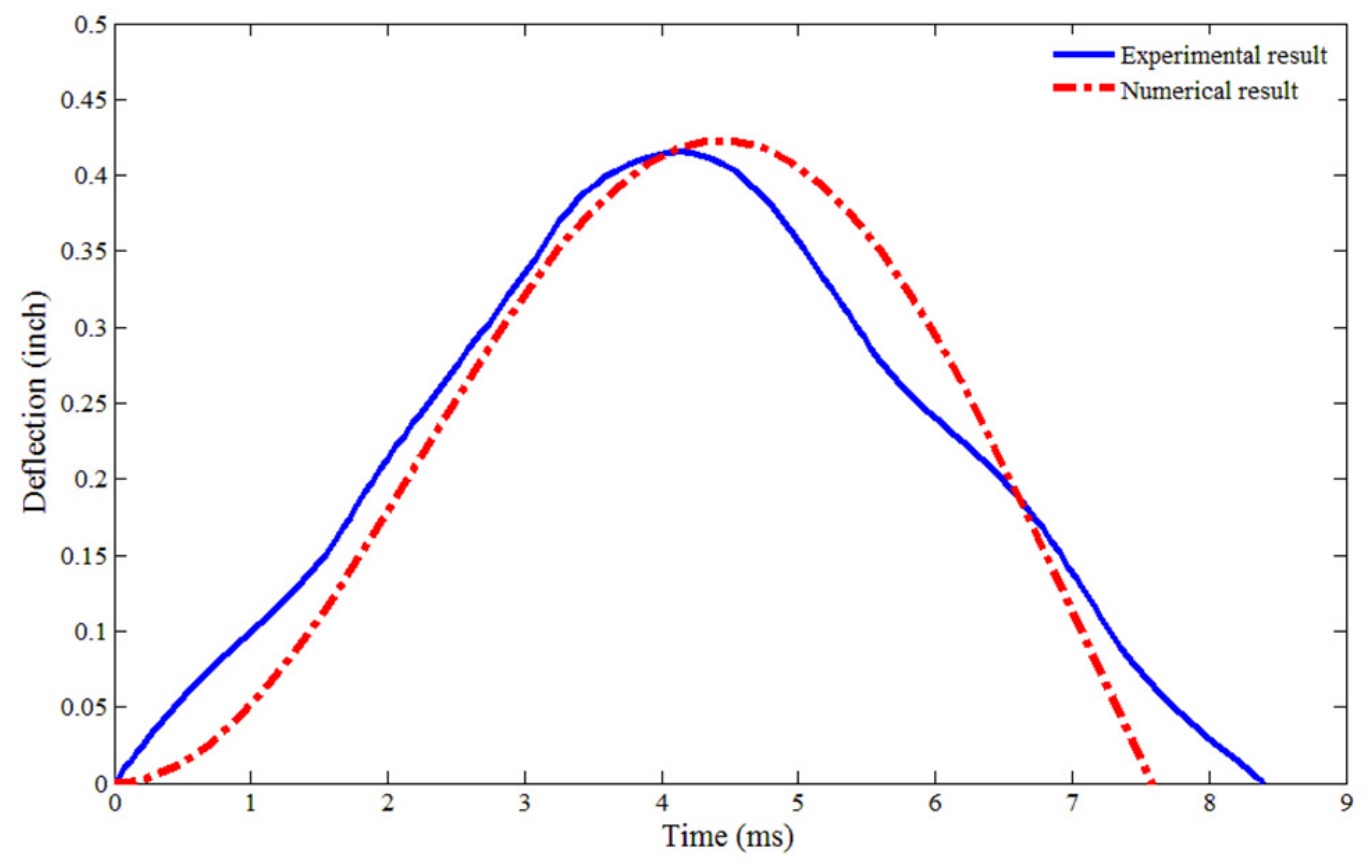

402 
Figure 14 Midpoint deflection of the laminated glass under the medium intensity blast loading in the first deflection peak region

405

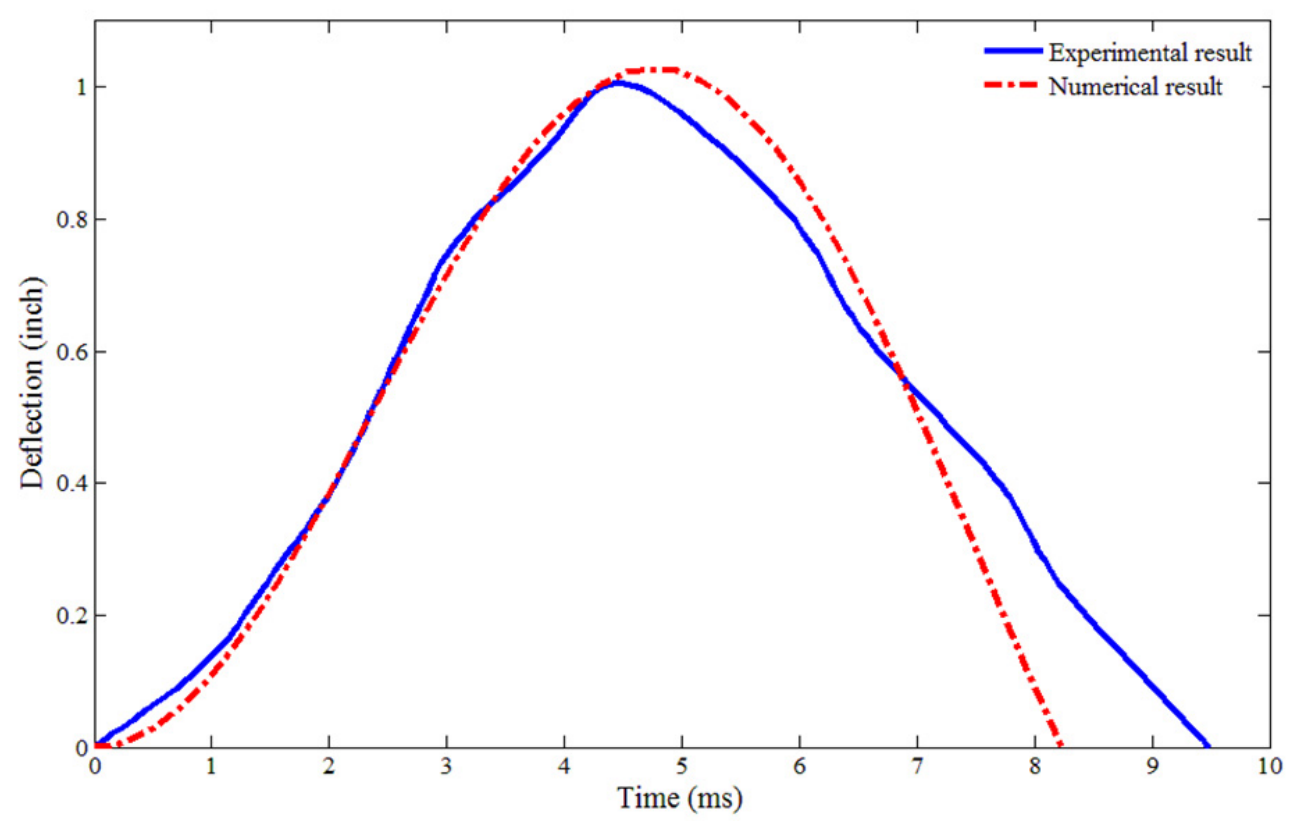

Figure 15 Midpoint deflection of the laminated glass under the high intensity blast loading in the first deflection peak region

\section{Laminated glass failure analysis}

$$
\sigma_{x}=\frac{E_{a v g}}{1-v_{a v g}^{2}}\left(\varepsilon_{x}+v_{a v g} \varepsilon_{y}\right)
$$

$$
\begin{aligned}
& \sigma_{y}=\frac{E_{a v g}}{1-v_{a v g}^{2}}\left(v_{a v g} \varepsilon_{x}+\varepsilon_{y}\right) \\
& \tau_{x y}=G_{a v g} \gamma_{x y}
\end{aligned}
$$




$$
\begin{aligned}
& \sigma_{x}=-\frac{E_{a v g} z}{1-v_{a v g}^{2}}\left(\frac{\partial^{2} w}{\partial x^{2}}+v_{a v g} \frac{\partial^{2} w}{\partial y^{2}}\right) \\
& \sigma_{y}=-\frac{E_{a v g} z}{1-v_{a v g}^{2}}\left(v_{a v g} \frac{\partial^{2} w}{\partial x^{2}}+\frac{\partial^{2} w}{\partial y^{2}}\right) \\
& \tau_{x y}=-2 G_{a v g} z \frac{\partial^{2} w}{\partial x \partial y}
\end{aligned}
$$

where $E_{a v g}, v_{a v g}$ and $G_{a v g}$ are the average Young's modulus, Poisson's ratio and shear modulus of 420 the laminated glass, respectively. These parameters can be calculated using the following 421 equations $[11,38]$

422

$$
\begin{aligned}
& E_{a v g}=\frac{E_{g} h_{g}+E_{c} h_{c}+E_{g} h_{g}}{h_{g}+h_{c}+h_{g}} \\
& v_{\text {avg }}=\frac{v_{g} h_{g}+v_{c} h_{c}+v_{g} h_{g}}{h_{g}+h_{c}+h_{g}} \\
& G_{a v g}=\frac{E_{g} h_{g}+2\left(1+v_{g}\right) G_{c} h_{c}+E_{g} h_{g}}{2\left(1+v_{g}\right)\left(h_{g}+h_{c}+h_{g}\right)}
\end{aligned}
$$

After obtaining the values of the deflection $w$, the stresses of the laminated glass can be 426 calculated using equation (17). And then the principal stresses of the laminated glass can be 427 calculated. According to the literature [33], the maximum principal stress always occurs at the 428 midpoint of the laminated glass. So the midpoint maximum principal stress history in the first 429 and second deflection peak regions (the first positive deflection peak region and the first negative 
430 deflection peak region) is calculated and compared with the tensile strength of tempered glass to

431 determine whether the laminated glass can survive when subjected to blast loading. This criterion

432 is proposed by Wei et al. and used in their research [11]. The reason for studying the principal

433 stress history in the first two deflection peak regions only is that according to equation (17),

434 stresses varies directly with the deflection w. From Fig. 7 and 9, it can be observed that

435 compared with the maximum deflection (the first deflection peak), the deflection after the first

436 two deflection peak regions is small, which means corresponding stresses/principal stresses are

437 small. Therefore, in this study, the principal stress history after the first two deflection peak

438 regions is not discussed. When subjected to the medium intensity blast loading, the midpoint

439 maximum principal stress histories of the inner glass surface (pressure impact surface) and the

440 outer glass surface (pressure non-impact surface) in the first two deflection peak regions are

441 calculated and plotted in Fig. 16, respectively.

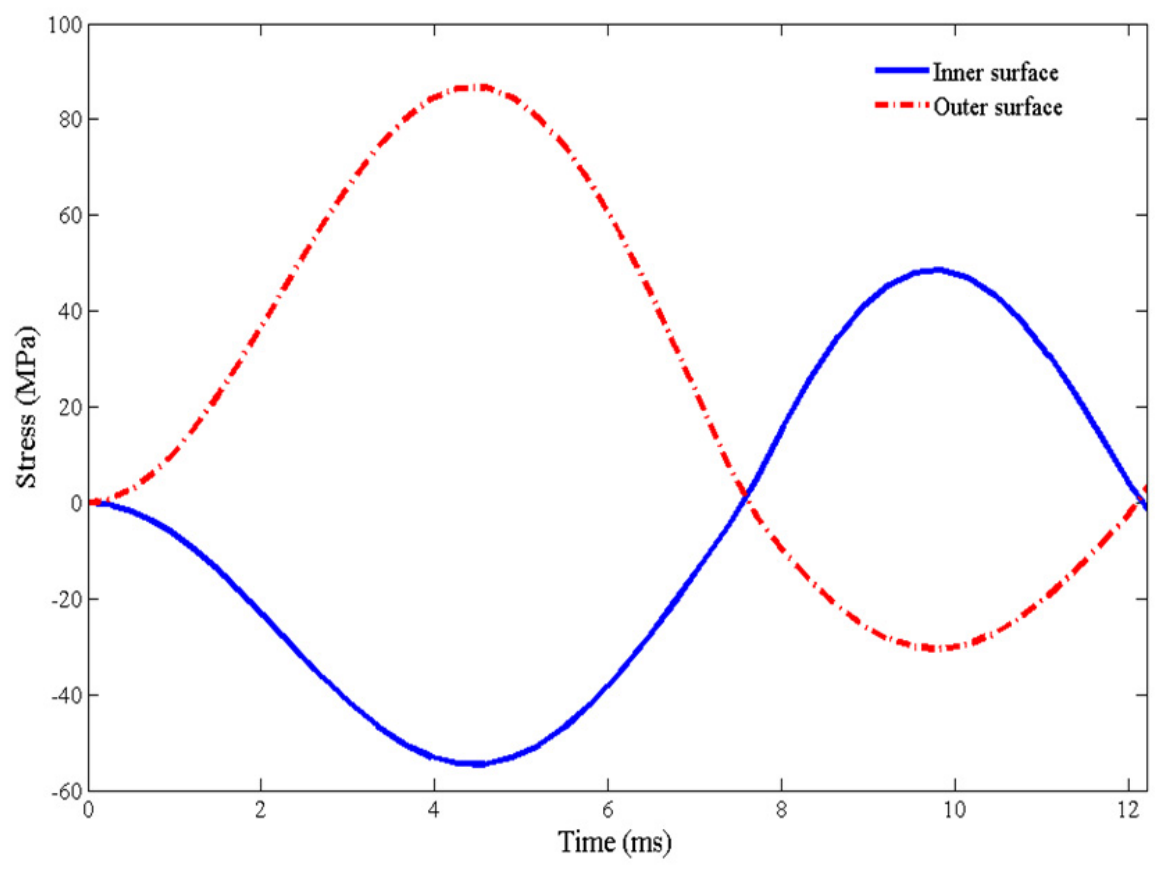

442

Figure 16 Midpoint maximum principal stress history under the medium intensity blast loading 
Fig. 16 illustrates that for the inner surface, the maximum compression stress is about 55

$447 \mathrm{MPa}$ and the maximum tensile stress is about $50 \mathrm{MPa}$; for the outer surface, the maximum 448 compression stress is about $31 \mathrm{MPa}$ and the maximum tensile stress is about $85 \mathrm{MPa}$. The tensile 449 strength of tempered glass is usually above $175 \mathrm{MPa}$ (sometimes above $200 \mathrm{MPa}$ ), and its 450 compressive strength is much higher than its tensile strength [39-42]. Therefore, according to the 451 stress analysis, the laminated glass will survive when subjected to the medium intensity blast 452 loading. This result has been proven by the experimental result.

453 When subjected to the high intensity blast loading, the midpoint maximum principal stress 454 histories of the inner surface and the outer surface in the first two deflection peak regions are 455 shown in Fig. 17. From Fig. 17, it can be observed that the outer glass layer first experiences its 456 maximum tensile stress of around $200 \mathrm{MPa}$, which is close to but not beyond the tensile strength 457 of tempered glass. Then, the inner glass layer experiences its maximum tensile stress of $\sim 95$ $458 \mathrm{MPa}$, which is lower than the tensile strength of tempered glass. Thus, the laminated glass will 459 survive exposure to the high intensity blast loading. This result corresponds with the 460 experimentally observed result. 


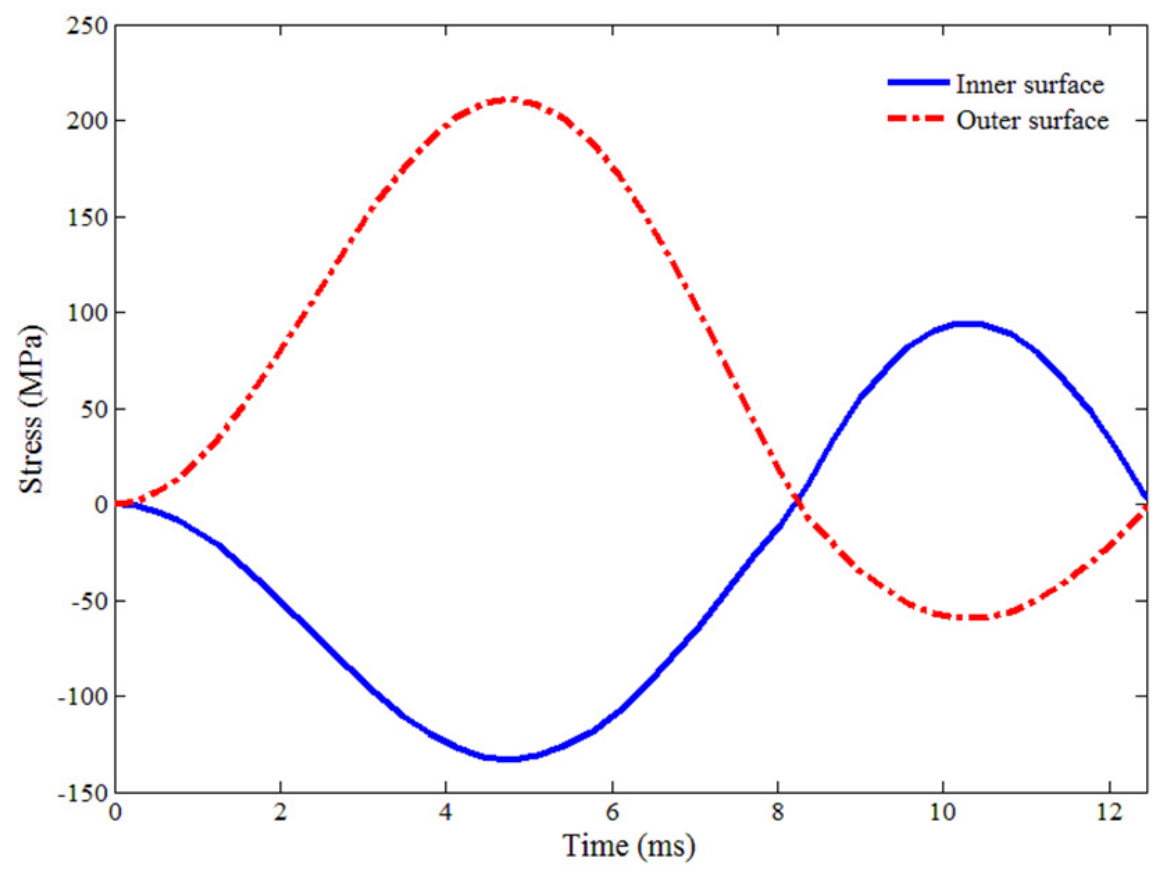

462

Figure 17 Midpoint maximum principal stress history under the high intensity blast loading

\subsection{Conclusions}

A novel laminated glass using a transparent glass fiber-reinforced composite interlayer has been developed. The light transmittance of the laminated glass is above $60 \%$ over a wavelength range of $482 \mathrm{~nm}$ to $900 \mathrm{~nm}$ and the highest transmittance is $84.4 \%$ when the wavelength is 577 $\mathrm{nm}$. The appearance of the laminated glass shows it has good transparency. experiments and a model-based simulation. The predicted dynamic response, in terms of the

473 midpoint deflection, agrees fairly well with the experimentally measured results under both 474 medium and high intensity blast loading. Stress analysis and experimental results both show that 475 the new laminated glass panel with a transparent glass fiber reinforced polymer interlayer can 476 survive under medium and high intensity blast loading. 


\section{Acknowledgement}

479 The financial support of the Department of Homeland Security Science and Technology

480 Directorate (DHS S\&T) under award numbers 2008-ST-108-000001 and HSHQDC-10-C-

$481 \quad 00138 \mathrm{CS}$ and NSF under award number 0324296 are gratefully acknowledged.

482

483

484

485

486

487

488

489

490

491

492

493

494

495

496

497

498

499

500

501

502

503

504

505

506

507

508

509

510

511

512

513

514 


\section{REFERENCES}

516 [1] Kamhine, A., Shapira, N., and Geva, R., 2000, "Terrorist bomb threat and the response in the 517 police bomb disposal division", Innovation Exchange, No. 8, pp.27-30.

518 [2] Little, R.G., 2005, "Risk management and protection strategies for buildings and facilities", 519 Journal of Urban Technology, 12, No. 2, pp.71-85.

520 [3] Kosal, M.E., 2006, "Terrorism targeting industrial chemical facilities: strategic motivations 521 and the implications for US security", Studies in Conflict \& Terrorism, 29, No. 7, pp.719-751.

522 [4] Zhang, X.H., Hao, H., and Ma, G.W., 2013, "Parametric study of laminated glass window 523 response to blast loads", Engineering Structures, 56, pp.1707-1717.

524 [5] Kumar, P., and Shukla, A., 2011, "Dynamic response of glass panels under shock loading", 525 Journal of Non-Crystalline Solids, 357, No. 24, pp.3917-3923.

526 [6] Lumantarna, R., Ngo, T., Mendis, P., and Lam, N., 2007, "Behaviour and modelling of 527 glazing components under full-scale blast tests in woomera", Proceedings of the 2007 RNSA 528 Security Technology Conference, pp.299-309.

529 [7] Hidallana-Gamage, H.D., Thambiratnam, D.P., and Perera, N.J., 2013, "Computational 530 analysis of laminated glass panels under blast loads: a comparison of two dimensional and three 531 dimensional modelling approaches", The International Journal of Engineering and Science, 2, 532 No.8, pp.69-79。

533 [8] Khanna, S.K., 2011, "Lightweight, thin blast-resistant glass developed", Advanced 534 Composites Bulletin, No. 2, pp.8.

535 [9] Magro, D.T., 1993, "Development of a decision method for glass windows under blast 536 loadings", DTIC Document.

537 [10] Zhang, X.H., Hao, H., and Ma, G.W., 2013, "Laboratory test and numerical simulation of 538 laminated glass window vulnerability to debris impact", International Journal of Impact 539 Engineering, 55, pp.49-62.

540 [11] Wei, J., and Dharani, L.R., 2006, "Response of laminated architectural glazing under blast 541 loading", Internaitonal Journal of Impact Engineering, 32, No. 12, pp.2032-2047.

542 [12] Norville, H.S., and Conrath, E.J., 2006, "Blast-resistant glazing design", Journal of 543 Architectural Engineering, 12, No. 3, pp.129-136.

544 [13] Weggel, D.C., and Zapata, B.J., 2008, "Laminated glass curtain walls and laminated glass 545 lites under low-level blast loading", Journal of Structural Engineering, 134, No. 3, pp.466-477.

546 [14] Hidallana-Gamage, H.D., Thambiratnam, D.P., and Perera, N.J., 2014, "Numerical modelling and analysis of the blast performance of laminated glass panels and the influence of 548 material parameters", Engineering Failure Analysis, 45, pp.65-84.

549 [15] Duser, A., Jagota, A., and Bennison, S., 1999, "Analysis of glass/polyvinyl butyral laminates 550 under uniform pressure", Journal of Engineering Mechanics, 125, No. 4, pp.435-442. 
[16] Hooper, P., Arora, H., and Dear, J., 2010, "Blast and impact resistance of laminated glass structures", Proceedings of the IMPLAST 2010 conference, pp.1-8.

[17] Pantelides, C.P., Horst, A.D., and Minor, J.E., 1992, "Post-breakage behavior of architectural glazing in Windstorms", Journal of Wind Engineering and Industrial Aerodynamics, 44, No. 1-3, pp.2425-2435.

[18] Tao, Z.X., Zhang, Q.L., Chen, J., and Xie, B.Y., 2010, "Experimental study on mechanical behavior of laminated glass's PVB Film", Advanced Materials Research, 168-170, pp.17565581761.

559 [19] Norville, H.S., King, K.W., and Swofford, J.L., 1998, "Behavior and strength of laminated glass", Journal of Engineering Mechanics, 124, No. 1, pp.46-53.

[20] Jawaid, M., Khalil, H., Bakar, A.A., and Khanam, P.N., 2011, "Chemical resistance, void content and tensile properties of oil palm/jute fibre reinforced polymer hybrid composites", Materials \& Design, 32, No. 2, pp.1014-1019.

[21] Ramesh, M., Palanikumar, K., and Hemachandra, R.K., 2012, "Mechanical property evaluation of sisal-jute-glass

[22] Ho, M.P., and Lau, K.T., 2012, "Design of an impact resistant glass fibrelepoxy composites using short silk fibres", Materials \& Design, 35, No. 2, pp.664-669.

[23] Khanna, S.K., 2014, "Transparent composites and laminates and methods for making", US Patent 8753464 B2.

[24] ASTM, 2007, "Standard test method for tensile properties of polymer matrix composite materials", West Conshohocken, PA: ASTM International.

[25] ASTM, 2007, "Standard test method for in-plane shear response of polymer matrix composite materials by tensile test of a $\pm 45^{\circ}$ laminate", West Conshohocken, PA: ASTM International.

[26] Smith, P.D., and Hetherington, J.G., 1994, "Blast and ballistic loading of structures", Oxford, UK: Butterworth-Heinemann.

[27] Kinney, G.F., and Graham, K.J., 1985, "Explosive shocks in air", Berlin and New York: Springer-Verlag.

[28] Baker, W.E., 1973, "Explosions in air", Austin, TX: University of Texas Press Austin.

[29] Pothan, L.A., Mai, Y., Thomas, S., and Li, R., 2008, "Tensile and flexural behavior of sisal fabric/polyester textile composites prepared by resin transfer molding technique", Journal of Reinforced Plastics and Composites, 27, No. 16-17, pp.1847-1866.

584 [30] Olson, J.R., Day, D.E., and Stoffer, J.O., 1992, "Fabrication and mechanical properties of 585 an optically transparent glass fiber/polymer matrix composite", Journal of Composite Materials, 586 26, No. 8, pp.1181-92.

587 [31] Iba, H., Chang, T., and Kagawa, Y., 2002, "Optically transparent continuous glass fibre588 reinforced epoxy matrix composite: fabrication, optical and mechanical properties", Composite 589 Science and Technology, 62, No. 15, pp.2043-2052. 
590 [32] Pai, P.F., 2007, "Highly flexible structures: modeling, computation, and experimen-tation", 591 Reston, VA: American Institute of Aeronautics \& Astronautics.

592 [33] Wei J., 2004, "Dynamics and failure analysis of architectural glazing under blast loading", 593 Rolla, MO: University of Missouri.

594 [34] Ugural, A.C., 1981, "Stresses in plates and shells", New York, NY: McGraw Hill.

595 [35] Barre, S., Chotard, T., and Benzeggagh, M., 1996, "Comparative study of strain rate effects 596 on mechanical properties of glass fibre-reinforced thermoset matrix composite", Composites 597 Part A: Applied Science and Manufacturing, 27, No. 12, pp.1169-1181.

598 [36] Kazanc1, Z., 2011, "Dynamic response of composite sandwich plates subjected to time599 dependent pressure pulses", International Journal of Non-Linear Mechanics, 46, No.5, pp.807600817.

601 [37] Kazanc1, Z., and Mecitoglu, Z., 2008, "Nonlinear dynamic behavior of simply supported 602 laminated composite plates subjected to blast", Journal of Sound and Vibration, 317, No.3, 603 pp.883-897.

604 [38] Vinson, J.R., 1999, "The behavior of sandwich structures of isotropic and composite 605 materials", Boca, Raton: CRC Press.

606 [39] Kalluri, R.S., 2007, "Failure of transparent polymer composite laminated glass panels 607 under impact loading", Columbia, MO: University of Missouri.

608 [40] Mencik, J., 1992, "Strength and fracture of glass and ceramics", New York, NY: Elsevier.

609 [41] Veer, F., Zuidema, J., Bos, F., and Romein, T., 2005, "The strength and fracture behaviour 610 of annealed and tempered float glass", Proceedings of the 9th International Conference on 611 Architectural and Automotive Glass (GPD), pp.1-6.

612 [42] National Glass, "Glass Manufacture", http://www.nationalglass.com.au/catalogues/NGP 613 _Section_20.pdf 